Alternating Gradient Synchrotron Department

Relativistic Heavy Ion Collider Project

BROOKHAVEN NATIONAL LABORATORY

Upton, New York 11973

Spin Note

AGS/RHIC/SN No. 055

\title{
Overcoming the Intrinsic Spin Resonance using Resonance Island created by RF Dipole*
}

M. Bai, S.Y. Lee

Department of Physics, Indiana University, Bloomington, IN 47405

H. Huang, T. Roser and M. Syphers

AGS Department, Brookhaven National Laboratory, NY 11973

May 5, 1997 


\title{
Overcoming the Intrinsic Spin Resonance using Resonance Island created by RF Dipole*
}

\author{
M. Bai, S. Y. Lee \\ Department of Physics, Indiana University, Bloomington, IN 47405 \\ H. Huang, T. Roser and M. Syphers \\ AGS Department, Brookhaven National Laboratory, NY 11973 \\ May 5,1997
}

\begin{abstract}
A method of overcoming intrinsic spin resonances by transporting polarized beam into a resonance island created by if dipoles for adiabatic spin flip is discussed. Specific parameters needed in achieving polarized beam acceleration in the AGS are estimated. Numerical simulations with the AGS parameters are performed to verify the theoretical estimation.
\end{abstract}

\footnotetext{
*The work is supported in part by the US Department of Energy
} 


\section{Introduction}

Spin motion in synchrotrons obeys the Thomas-BMT equation [1]. For a planar synchrotron with a vertical guide field, the polarization vector precesses $G \gamma$ turns per orbital revolution, where $G=1.792847386$ is the anomalous $g$-factor for protons, $\gamma$ is the Lorentz factor. Thus $G \gamma$ is called the spin tune. In high energy accelerators, beam stability requires strong focusing quadrupoles. The non-vertical magnetic fields can perturb the spin away from the vertical direction. In particular, a spin depolarization resonance occurs when the spin tune is equal to the tune of spin perturbing fields.

During acceleration toward high energy, polarized beams encounter many depolarization resonances $[2,3]$. Two major types of spin depolarization resonance exist in synchrotrons, i.e., the imperfection and the intrinsic resonances. The imperfection spin resonance is created by the vertical closed orbit deviation from the center of quadrupoles, and the intrinsic spin resonance is produced by the vertical betatron oscillations. The resonance condition is given by

$$
G \gamma= \begin{cases}n & \text { for imperfection spin resonance, } \\ k P \pm m \nu_{z} & \text { for intrinsic spin resonance }\end{cases}
$$

where $k, m$ and $n$ are integers, $P$ is the number of superperiods, and $\nu_{z}$ is the vertical betatron tune. For polarized protons in the AGS, we have $P=12$, and $\nu_{z} \approx 8.8$. Along with these two major spin resonances, depolarization can also occur at the linear coupling resonance line (see e.g. [4]), and at the synchrotron sidebands of primary spin resonances [5].

Using the Thomas-BMT equation [1], the spin resonance strength is given by the Fourier amplitude of the spin perturbing fields in synchrotrons $[2,3]$, i.e.

$$
\epsilon_{K}=\frac{1}{2 \pi} \oint\left[(1+G \gamma) \frac{\Delta B_{x}}{B \rho}+i(1+G) \frac{\Delta B_{\|}}{B \rho}\right] e^{i K \theta} d s,
$$

where $\theta$ is the orbital bending angle, $s$ is the distance along the reference orbit, $\Delta B_{x}$ is the radial perturbing field, $\Delta B_{\|}$is the longitudinal perturbing field, and $B \rho$ is the magnetic rigidity of the beam.

A partial Siberian snake has been successfully employed to overcome imperfection resonances at the AGS, and the IUCF Cooler Ring [4]. On the other hand, overcoming intrinsic resonances, which are traditionally corrected by the tune jump method [6], remains to be a difficult task. The difficulty in the tune jump method is that it will also cause emittance growth resulting from non-adiabatic optical mismatch and particularly the non-adiabatic betatron oscillations induced by an off-centered closed orbit in the tune jump ferrite quadrupoles [7]. For attaining polarized proton collisions in the relativistic heavy ion collider (RHIC), the polarized beam in the AGS must be accelerated up to $G \gamma=47.5$ passing through many intrinsic resonances at $G \gamma=\nu_{z}, 24-\nu_{z}, 12+\nu_{z}, 36-\nu_{z}, 24+\nu_{z}, 48-\nu_{z}$ 
and $36+\nu_{z}$. Thus finding a scheme to overcome intrinsic spin resonances without causing emittance dilution is an important topic in accelerator physics.

This paper studies the feasibility of polarized proton acceleration through intrinsic spin resonances by using the betatron resonance islands generated by a radio frequency (rf) dipole field. Section II provides theoretical framework for the method. Numerical simulations and the AGS modeling are given in Section III, and the conclusion is given in Section IV.

\section{The theoretical framework}

When the spin tune of a polarized vector is uniformly accelerated through an isolated resonance with a resonance strength $\epsilon$, the ratio of the final polarization $P_{f}$ to the initial polarization $P_{i}$ is given by the Froissart-Stora formula [8]

$$
\frac{P_{f}}{P_{i}}=2 e^{-\frac{\pi|\epsilon|^{2}}{2 \alpha}}-1,
$$

where $\epsilon$ is the resonance strength, and

$$
\alpha=\frac{d\left(G \gamma-k P \mp \nu_{z}\right)}{d \theta}
$$

is the acceleration rate. Based on the Froissart-Stora formula, one might think that the polarization of the beam could be maintained by the adiabatic spin-flip through a strong spin resonance.

However, the intrinsic spin resonance strength is proportional to the betatron amplitude or the square root of the vertical betatron action for an intrinsic resonance. Since a beam of polarized protons is usually composed of particles with different betatron actions, the final polarization is obtained through the ensemble average of the Froissart-Stora formula over all particles with different betatron amplitudes. The final polarization after passing through an intrinsic resonance for a beam with Gaussian distribution becomes

$$
\left\langle\frac{P_{f}}{P_{i}}\right\rangle=\frac{1-W_{0}}{1+W_{0}},
$$

where $W_{0}=\pi\left|\epsilon_{0}\right|^{2} / \alpha$, and $\epsilon_{0}$ is the spin resonance strength for the particle with the rms betatron action $I_{\text {rms }}$ of the beam. Here the rms action of the beam is defined by the rms beam size with

$$
I_{\mathrm{rms}}=\frac{\sigma_{z}^{2}}{2 \beta_{z}},
$$

where $\sigma_{z}$ is the vertical rms beam size, and $\beta_{z}$ is the vertical betatron amplitude. This means that the adiabatic spin-flip by a slow acceleration through an intrinsic resonance cannot efficiently be used in polarized proton acceleration. Since 
particles at the center of the beam bunch with small actions do not flip their spin, partial beam depolarization is usually inevitable.

To circumvent this difficulty, we can give the beam a coherent betatron excitation so that all particles have large betatron actions and the resulting ensemble average of the final polarization is essentially the same as that obtained from the Froissart-Stora formula [8]. However, betatron oscillations of different particles with different betatron tunes can decohere rapidly. This will result in emittance growth and performance degradation.

A coherent betatron oscillation without emittance dilution could be maintained by creating a resonance island in the betatron phase space, generating a potential well to maintain the bunch transverse phase space profile. This idea can be achieved by a modulation of dipole, which can create $1: 1$ parametric resonance islands $[9,10]$ in the vertical phase space. This section discusses a method in producing 1:1 parametric resonance islands.

\subsection{Effect of $\mathrm{rf}$ dipole on betatron motion}

The vertical betatron equation of motion is given by

$$
z^{\prime \prime}+K_{z}(s) z=-\frac{\Delta B_{x}}{B \rho}
$$

where $z$ is the vertical betatron coordinate, the prime corresponds to the derivative with respect to the longitudinal coordinate $s, K_{z}(s)$ is the focusing function, and

$$
\Delta B_{x}=\Delta B(s) \cos \nu_{\mathrm{m}} \theta
$$

is the rf horizontal dipole field with $\nu_{\mathrm{m}}$ as the modulation tune given by $\nu_{\mathrm{m}}=$ $f_{\mathrm{rf}} / f_{0}$. Here $f_{\mathrm{rf}}$ is the rf frequency of the rf dipole field, and $f_{0}$ is the revolution frequency. The corresponding Hamiltonian is given by

$$
H=\frac{1}{2} z^{\prime 2}+\frac{1}{2} K_{z} z^{2}+\frac{\Delta B(s)}{B \rho} z \cos \nu_{\mathrm{m}} \theta
$$

Using the generating function $F_{1}$,

$$
F_{1}=-\frac{z^{2}}{2 \beta_{z}}\left(\tan \phi_{z}-\frac{\beta_{z}}{2}\right),
$$

where $\beta_{z}(s)$ is the betatron amplitude function, $\phi_{z}(s)$ is the new conjugate phase coordinate, the canonical transformation is given by

$$
z=\sqrt{2 \beta_{z} I_{z}} \cos \phi_{z}, \quad p_{z}=\alpha_{z} z+\beta_{z} z^{\prime}=-\sqrt{2 \beta_{z} I_{z}} \sin \phi_{z}
$$

Here $I_{z}$ and $\phi_{z}$ are conjugate action-angle phase space coordinates. The new Hamiltonian becomes

$$
H\left(I_{z}, \phi_{z}\right)=\frac{I}{\beta_{z}}+\frac{\Delta B}{B \rho} \sqrt{2 \beta_{z}} I_{z}^{\frac{1}{2}} \cos \phi_{z} \cos \nu_{\mathrm{m}} \theta
$$


where $s$ is still serving as the time variable.

Using the generating function

$$
F_{2}=\left(\phi_{z}-\mu_{z}+\nu_{z} \theta\right) J_{z}, \quad \mu_{z}(s)=\int_{0}^{s} \frac{d s}{\beta_{z}},
$$

where $J_{z}$ and $\psi_{z}=\phi_{z}-\mu_{z}(s)+\nu_{z} \theta$ are conjugate phase space coordinates, and using the orbit angle $\theta=s / R$ as the time variable, the new Hamiltonian becomes

$$
H\left(J_{z}, \psi_{z}\right)=\nu_{z} J_{z}+\frac{R \Delta B(s)}{B \rho} \sqrt{2 \beta_{z} J_{z}} \cos \left(\psi_{z}+\mu_{z}-\nu_{z} \theta\right) \cos \nu_{\mathrm{m}} \theta,
$$

where $R$ is the mean radius of the accelerator.

Since $\Delta B(s)$ and $\mu_{z}(s)-\nu_{z} \theta$ are periodic function of $\theta$, the Hamiltonian of Eq. (10) can be expanded in Fourier series:

$$
H\left(J_{z}, \psi_{z}\right)=\nu_{z} J_{z}+\frac{1}{2} \sum_{n} \sqrt{2 J_{z}}\left[C_{n+} e^{i\left(\psi_{z}-n \theta\right)}+C_{n-} e^{-i\left(\psi_{z}+n \theta\right)}\right] \cos \nu_{\mathrm{m}} \theta
$$

where we have

$$
\begin{aligned}
& C_{n+}=\frac{1}{2 \pi} \int_{0}^{2 \pi R} \frac{\Delta B(s)}{B \rho} \sqrt{\beta_{z}} e^{i\left(\mu_{z}-\nu_{z} \theta\right)} e^{i n \theta} d s, \\
& C_{n-}=\frac{1}{2 \pi} \int_{0}^{2 \pi R} \frac{\Delta B(s)}{B \rho} \sqrt{\beta_{z}} e^{-i\left(\mu_{z}-\nu_{z} \theta\right)} e^{i n \theta} d s .
\end{aligned}
$$

In particular, a localized if dipole will produce all integer harmonics in the Fourier expansion.

The resonance condition is given by $\nu_{\mathrm{m}}=n \pm \nu_{z}$. For example, $\nu_{z} \approx 8.8$, the resonance condition can be fulfilled by $\nu_{\mathrm{m}} \approx 0.2$ with $n=9$. At a resonance condition $\nu_{\mathrm{m}}=n \pm \nu_{z}$, we need to retain only the resonance term

$$
H\left(J_{z}, \psi_{z}\right) \approx \nu_{z} J_{z}+\frac{1}{2} \sqrt{2 J_{z}}\left|C_{\mathrm{eff}}\right| \cos \left(\psi_{z}-n \theta+\nu_{\mathrm{m}} \theta+\chi\right),
$$

where $\chi$ is the phase of the resonance strength, and

$$
\left|C_{\text {eff }}\right|=\frac{\Delta B \Delta L}{2 \pi B \rho} \sqrt{\beta_{z}}
$$

with the integrated if dipole field strength $\Delta B \Delta L=\int \Delta B(s) d s$.

We now transform the phase space into the resonance rotating frame by using the generating function

$$
F_{2}=\left(\psi_{z}-n \theta+\nu_{\mathrm{m}} \theta+\chi\right) J,
$$

and the new Hamiltonian becomes

$$
H(\psi, J)=\delta J+\frac{1}{2}\left|C_{\mathrm{eff}}\right| \sqrt{2 J} \cos \psi
$$


where $\delta=\nu_{z}-\left(m-\nu_{\mathrm{m}}\right)$ is the resonance proximity parameter, $J=J_{z}$ and $\psi=\psi_{z}-n \theta+\nu_{z} \theta+\chi$. Now, we add a nonlinear betatron detuning term to the Hamiltonian $H(\psi, J)$ (see Appendix A), i.e.,

$$
H(\psi, J)=\delta J+\frac{1}{2} \alpha_{z z} J^{2}+\frac{1}{2}\left|C_{\mathrm{eff}}\right| \sqrt{2 J} \cos \psi
$$

where the detuning parameter $\alpha_{z z}$ are generated by using octupole magnets or the second order effect of sextupole magnets.

The fixed points of the resonance Hamiltonian is given by

$$
\dot{J}=-\frac{\partial H}{\partial \psi}=0, \quad \dot{\psi}=\frac{\partial H}{\partial J}=0 .
$$

Equation (19) gives $\psi_{\mathrm{PP}}=0$ or $\pi$ and

$$
\delta+\alpha_{z z} J+\frac{1}{2} C_{\mathrm{eff}}(2 J)^{-\frac{1}{2}} \cos \psi_{z}=0
$$

Defining the parameter $w=\sqrt{2 J} \cos \psi_{\mathrm{pP}}$, where $\psi_{\mathrm{pP}}=0$ or $\pi$, the equation for the fixed point becomes

$$
w^{3}+\frac{2 \delta}{\alpha_{z z}} w+\frac{C_{\text {eff }}}{\alpha_{z z}}=0
$$

Letting the resonance strength parameter $a$ and the resonance proximity $b$ be

$$
a=2 \frac{C_{\mathrm{eff}}}{\alpha_{z z}}, \quad b=\frac{2 \delta}{\alpha_{z z}},
$$

Eq. (20) is simplified to

$$
w^{3}-b w+\frac{1}{2} a=0
$$

which has three solutions for $b \geq b_{b}=3\left(\frac{a}{4}\right)^{\frac{2}{3}}$ given by

$$
\begin{aligned}
& w_{1}=-\frac{2}{\sqrt{3}} b^{\frac{1}{2}} \cos \frac{\xi}{3}, \\
& w_{2}=\frac{2}{\sqrt{3}} b^{\frac{1}{2}} \sin \left(\frac{\pi}{6}-\frac{\xi}{3}\right), \\
& w_{3}=\frac{2}{\sqrt{3}} b^{\frac{1}{2}} \sin \left(\frac{\pi}{6}+\frac{\xi}{3}\right),
\end{aligned}
$$

with $\xi=\arctan \sqrt{\left(b / b_{b}\right)^{3}-1}$. Here $w_{1}$ and $w_{2}$ are stable fixed points (SFP) and $w_{3}$ is the unstable fixed point (UFP). 
Using the normalized coordinates with

$$
h_{i}=\sqrt{\frac{3}{b_{b}}} w_{i}
$$

for $i=1,2,3$, the intercepts of the separatrix with the betatron coordinate are given by

$$
\begin{aligned}
& h_{a}=-h_{3}-\frac{2}{\sqrt{h_{3}}} \\
& h_{b}=-h_{3}+\frac{2}{\sqrt{h_{3}}} .
\end{aligned}
$$

Figure 1 shows the fixed points of resonance islands and the intercepts of the separatrix with a phase space coordinate as a function of the resonance proximity parameter $b / b_{b}$.

\subsection{Requirements for the bunched beam manipulation}

Assuming a synchrotron with dynamical aperture $D_{0}$, the stability condition of particle motion is given by

$$
\sqrt{\beta_{z}} w_{a} \leq D_{0}
$$

To fit the beam bunch with a $95 \%$ normalized emittance $\varepsilon_{N}$ into the resonance island, the additional condition is given by

$$
\sqrt{\beta_{z}}\left|w_{a}-w_{b}\right| \geq \sqrt{6 \frac{\varepsilon_{N} \beta_{z}}{\pi \gamma}}
$$

where the factor of 6 depicts the fact that the island is large enough to encompass $6 \sigma_{z}$. In order to put the beam bunch into the center of the resonance island, the angular kick $\theta_{k}$ needed to transport the beam bunch from the SFP $w_{2}$ to the SFP $w_{1}$ is given by

$$
\beta_{z k} \theta_{k}=\sqrt{\beta_{z k}}\left|w_{1}-w_{2}\right|
$$

or

$$
\delta=\frac{1}{2} \alpha_{z z} \beta_{z k} \theta_{k}^{2}=\alpha_{z z} I_{\mathrm{sfp}},
$$

where $\beta_{z k}$ is the betatron amplitude function at the kicker location. Alternately, the beam bunch can be transported into the outer island by adiabatically ramping the betatron tune. Equation (31) provides the tune ramp needed to generate coherent betatron oscillation with a coherent betatron action $I_{\text {sfp. }}$.

Following an angular kick $\theta_{k}$, the Gaussian distribution function becomes

$$
\rho\left(z, p_{z}\right)=\frac{1}{2 \pi \sigma_{z}^{2}} \exp \left\{-\frac{z^{2}+\left(p_{z}+\beta_{z k} \theta_{k}\right)^{2}}{2 \sigma_{z}^{2}}\right\}
$$


where the rms beam size is $\sigma_{z}=\sqrt{\beta_{z} \varepsilon_{\mathrm{rms}}}$, and

$$
z=\sqrt{2 \beta_{z} J} \cos \psi, \quad p_{z}=-\sqrt{2 \beta_{z} J} \sin \psi
$$

When the kicked beam is accelerated through an intrinsic spin resonance, the final polarization, given by the ensemble average of the Froissart-Stora formula over the beam distribution, becomes

$$
\left\langle\frac{P_{f}}{P_{i}}\right\rangle=\frac{2}{1+\frac{\pi\left|\epsilon_{0}\right|^{2}}{\alpha}} \exp \left\{-\frac{\left(\frac{\left(\beta_{z k} \theta_{k}\right)^{2}}{2 \sigma_{z}^{2}}\right) \frac{\pi\left|\epsilon_{0}\right|^{2}}{\alpha}}{1+\frac{\pi\left|\epsilon_{0}\right|^{2}}{\alpha}}\right\}-1
$$

where $\epsilon_{0}$ is the resonance strength of rms particle with action $I_{\text {rms. }}$. In order to achieve a $99 \%$ spin flip, the kicker angle requirement is

$$
\beta_{z k} \theta_{k} \geq 3.3\left(1+\frac{\alpha}{\pi\left|\epsilon_{0}\right|^{2}}\right)^{1 / 2} \sigma_{z}
$$

Clearly, the method is not applicable to weak spin resonances where $\pi\left|\epsilon_{0}\right|^{2} / \alpha \ll 1$.

\section{Application to the AGS Polarized Beam Ac- celeration}

The AGS lattice consists of combined function magnets with $P=12$ superperiods, $\nu_{z}=8.8$, and $\beta_{z, \max }=22 \mathrm{~m}$. The polarized proton acceleration rate is normally $\alpha=4.5 \times 10^{-5}$. The normalized emittance of polarized proton beam is about $10 \sim 20 \pi \mathrm{mm}$-mrad. Table 1 lists the spin resonance strength $\epsilon_{0}$ for the rms particle with normalized $2.5 \pi \mathrm{mm}-\mathrm{mrad}$ emittance [6]. This corresponds to a beam of $15 \pi \mathrm{mm}$-mrad $95 \%$ emittance. The parameter $W_{0}=\pi\left|\epsilon_{0}\right|^{2} / \alpha$ is listed in the third column. The 4 th column is the beam size with $2.5 \pi \mathrm{mm}$-mrad normalized rms emittance. The required $99 \%$ spin flip kick amplitude $\beta_{z k} \theta_{k}$ is shown in the fifth column, and the required minimum half-aperture is listed in the sixth column, where the aperture is given by $D_{0, \min }=\beta_{z k} \theta_{k}+3 \sigma_{z}$. Clearly, the present method can only be used to overcome strong spin resonances at $0+\nu_{z}$, $12+\nu_{z}, 36-\nu_{z}$ and $36+\nu_{z}$. Fortunately, weak spin resonances at $24-\nu_{z}, 24+\nu_{z}$ and $48-\nu_{z}$ do not require correction for a properly tuned lattice with a small emittance beam.

The proximity parameter is chosen such that the resulting outer SFP is large enough to reach a $99 \%$ spin flip. The rf modulation field strength is chosen such that the island size is large enough to encompass the entire bunch. The 7th column of Table 1 lists the required integrated field strength of the rf dipole in Gauss-meters, which is obtained by solving Eq. (29) with a $95 \%$ normalized emittance at $\epsilon_{N}=15 \pi \mathrm{mm}-\mathrm{mrad}$ and a typical detuning parameter $\alpha_{z z}=1000$ $(\pi-\mathrm{m})^{-1}$. Although a larger $\alpha_{z z}$ parameter is preferable for the stability of the beam near the 1:1 parametric resonance, we will discuss the effect of rf dipole in the small detuning limit in next section. In the following, we discuss other issues associated with polarized proton acceleration using the if dipole scheme. 
Table 1: Intrinsic resonance strengths of the AGS

\begin{tabular}{|l|l|l|l|l|l|l|}
\hline$G \gamma$ & $\epsilon(2.5 \pi)$ & $\pi\left|\epsilon_{0}\right|^{2} / \alpha$ & $\begin{array}{l}\sigma_{z} \\
(\mathrm{~mm})\end{array}$ & $\begin{array}{l}\beta_{z k} \theta_{k} \\
(\mathrm{~mm})\end{array}$ & $\begin{array}{l}D_{0, \min } \\
(\mathrm{mm})\end{array}$ & $\begin{array}{l}(B L)_{\mathrm{rf}} \\
(\mathrm{Gm})\end{array}$ \\
\hline $0+\nu_{z}$ & 0.0075 & 3.9 & 3.3 & 12.4 & 25 & 8 \\
$24-\nu_{z}$ & 0.0003 & 0.006 & 2.5 & 107 & - & - \\
$12+\nu_{z}$ & 0.0029 & 0.6 & 2.2 & 11.8 & 18 & 5 \\
$36-\nu_{z}$ & 0.0062 & 2.7 & 1.9 & 7.3 & 14 & 5 \\
$24+\nu_{z}$ & 0.0005 & 0.02 & 1.7 & 40 & - & - \\
$48-\nu_{z}$ & 0.00077 & 0.04 & 1.6 & 27 & - & - \\
$36+\nu_{z}$ & 0.0134 & 12.5 & 1.5 & 5.1 & 10 & 4 \\
\hline
\end{tabular}

\subsection{Numerical simulations}

There are three methods to transport the beam bunch into the 1:1 parametric outer resonance island. The first method uses a single turn dipole kicker to transport the beam from the inner fixed point $w_{2}$ into the outer fixed point $w_{1}$ [9]. The second method employs slow quadrupoles to change the vertical betatron tune in order to transport the beam bunch along the $w_{1}$ SFP from the $b / b_{b}<1$ region to the $b / b_{b}>1$ region [10]. The third method adiabatically ramps the rf dipole field strength at $b / b_{b}<1$ region in order to increase the amplitude of coherent betatron oscillations.

\subsubsection{Coherent betatron oscillation with a dipole kick}

Using a dipole kick, the beam bunch is transported from the inner attractor $w_{2}$ to the outer attractor $w_{1}$ by a pulsed dipole. The bottom plot of Fig. 2 shows the phase space ellipse of a beam bunch with a $95 \%$ normalized emittance of $20 \pi \mathrm{mm}$-mrad being transported into the outer island. The top plot shows the Poincare surface of section at the end of 500 orbital revolutions. Note here that the mismatch of the beam ellipse can result in a substantial emittance dilution. By observing the mismatch of the equipotential contour of the inner and outer SFPs, we find that emittance dilution is inevitable for the kick method.

\subsubsection{Coherent betatron oscillation with slow tune ramp}

Using slow quadrupole magnets to vary the tune, the center of the bunch can be adiabatically transported along the SFP $w_{1}$ (see the bottom plot of Fig. 1 of Ref. [10]). An example of a tune ramp is shown in Fig. 3. The top left plot shows an initial distribution along with its potential contour, where the rf dipole is on without any tune ramp. The top right plot shows the beam profile after the tune is changed by 0.02 in 600 turns. The tune is held at this value to cross 
the intrinsic spin resonance for another 600 turns. At the end of 1200 turns, the Poincaré surface of section is shown in the bottom left plot. After crossing the spin resonance, the tune is ramped back to its original value. The final Poincaré surface of section for the beam bunch is shown in the bottom right plot. Note here that the adiabatic process renders a minimum emittance growth for the bunch beam manipulation. Our numerical simulations show that the minimum number of turns for attaining adiabatic transport of the beam bunch is about 500 turns.

\subsubsection{Coherent Betatron oscillations in the zero detuning limit}

When the nonlinear detuning is reduced, the amplitude of the 1:1 parametric resonance becomes much more sensitive to the proximity parameter $\delta$. A small change of the $\delta$ parameter, can result in a large change in the parameter $b / b_{b}$ in Fig. 1. Figure 5 shows the fixed points in $\mathrm{mm}$ at the location of $\beta_{z}=22 \mathrm{~m}$ for the parameter $\alpha_{z z}=0,100$, and $500 \mathrm{~m}^{-1}$ respectively. Thus the outer SFP of the 1:1 parametric resonance may lie outside the dynamical aperture (about $38 \mathrm{~mm}$ ) in the small detuning parameter case. The procedure to induce an enhanced betatron oscillation is to operate the rf dipole with a proximity parameter of about 0.01 in the parametric region $b / b_{b} \leq 1$. Then the rf dipole modulation amplitude is adiabatically increased so that the beam follows the SFP of the 1:1 resonance.

The integrated rf dipole field strength in the zero detuning limit is related to the coherent betatron amplitude $z_{\text {coh }}$ by

$$
\left.\int \Delta B d s\right|_{\mathrm{rf}}=4 \pi(B \rho) \delta z_{\mathrm{coh}} / \beta
$$

where $z_{\text {coh }}$ is the fixed point of the Hamiltonian in Eq. (17). The fifth column in Table 2 shows the integrated of dipole strength needed to achieve the required amplitude of the coherent motion $z_{\text {coh }}=\beta_{z} \theta_{k}$ of Table 1 . Numerical simulations showed that the adiabaticity can be maintained easily when the modulation amplitude is varied. The resulting emittance growth factor is listed in the sixth column of Table. 2.

\subsection{Physics of coherent $r f$ spin resonance}

Since the tune of the coherent vertical betatron oscillations induced by the rf dipoles at a modulation tune $\nu_{\mathrm{m}}$ in AGS is $9-\nu_{m}$, coherent spin resonances are located at $G \gamma=k P \pm\left(9-\nu_{\mathrm{m}}\right)$. The coherent spin resonance is identical to the intrinsic spin resonance resulting from spin kicks due to quadrupoles in the synchrotron. At the same time, the if dipole also generates if spin resonance located at

$$
K_{\mathrm{rf}}=n \pm \nu_{\mathrm{m}}
$$


Table 2: Rf dipole strength is listed together with the parameters of the strong intrinsic resonances in the AGS. The last column shows the emittance growth obtained in numerical simulations. The amplitude of the if dipole was ramped up during 1000 turns left at the maximum value for 2000 turns and then ramped down over again 1000 turns. A betatron tune spread of \pm 0.002 due to some residual non-zero chromaticity and a residual detuning parameter of $40 \mathrm{~m}^{-1}$ was also included.

\begin{tabular}{|r|r|r|r|r|c|}
\hline$G \gamma$ & $\begin{array}{r}B \rho \\
(\mathrm{Tm})\end{array}$ & $\epsilon(2.5 \pi)$ & $\begin{array}{r}z_{\text {coh }} \\
{[\mathrm{mm}]}\end{array}$ & $\begin{array}{r}\int \Delta B d s \\
{[\mathrm{Gm}]}\end{array}$ & $\begin{array}{c}\text { emittance } \\
\text { growth ratio }\end{array}$ \\
\hline $0+\nu_{z}$ & 15.1 & 0.0075 & 12.4 & 11. & 1.07 \\
$12+\nu_{z}$ & 36.2 & 0.0029 & 11.8 & 24. & 1.07 \\
$36-\nu_{z}$ & 47.4 & 0.0062 & 7.3 & 20. & 1.00 \\
$36+\nu_{z}$ & 78.2 & 0.0134 & 5.1 & 23. & 1.01 \\
\hline
\end{tabular}

The induced rf resonance strength is given by

$$
\epsilon_{\mathrm{rf}}=\frac{1+G \gamma}{2 \pi} \frac{(B L)_{\mathrm{rf}}}{B \rho} .
$$

Since the rf dipole field needed for polarized beam operation is very small (see Tables 1 and 2), the rf induced spin resonance has little effects on spin motion.

An alternative scheme of spin flip is to generate large if spin resonance so that the spin motion near the intrinsic spin resonance will adiabatically follow the spin closed orbit of the rf spin resonance. If we choose the rf spin resonance location $K_{\mathrm{rf}}$ near the intrinsic spin resonance, the spin motion will be dominated by the rf resonance. To reach an $\mathrm{rf}$ spin resonance strength of $\epsilon_{\mathrm{rf}}=0.025$, the rf dipole integrated field strength is about 2500 Gauss-meters. Such a high field if dipole is hard to achieve.

The basic idea of attaining a large coherent intrinsic spin resonance is to excite coherent betatron oscillations of the beam. When the rf dipole field is adiabatically turned on, the synchronous particle with a zero betatron action is adiabatically transported to the SFP of the 1:1 parametric resonance, which has a large coherent betatron action. Particles with nonzero actions orbit around the SFP at the island tunes given by

$$
\nu_{\text {island }} \approx \alpha_{x z} J_{x}+\alpha_{z z} J_{z}+\cdots,
$$

where $J_{x}$ and $J_{z}$ are the betatron actions of a particle relative to the center of the bunch. Thus particles with nonzero initial betatron actions will performs coherent betatron oscillations with amplitude and phase modulations around the SFP of the 1:1 parametric resonance. The amplitude modulation is averaged out 
to give a coherent spin resonance strength, while the phase modulation can create many island sidebands located at

$$
K=k P \pm\left(9-\nu_{\mathrm{m}}\right) \pm \ell \nu_{\text {island }}
$$

where $\ell=$ integer. Since the island tune is small, overlapping sidebands can be summed to obtain a single coherent spin resonance.

A beam bunch is also composed of off-momentum particles. If the chromaticity is not zero, the betatron tune of the particle with an off momentum value $\Delta p / p_{0}$ is given by

$$
\nu_{z, \mathrm{off}}=\nu_{z}+C_{z} \frac{\Delta p}{p_{0}} .
$$

When the rf dipole is adiabatically excited at the modulation tune $\nu_{\mathrm{m}}$, all particles with an off momentum $\Delta p / p_{0}$ will execute coherent betatron oscillations around a SFP that differs from the SFP of the synchronous particle. The polarization of the beam after passing through the intrinsic spin resonance is the ensemble average of the beam particles.

The spin tune modulation of an off momentum particle produces synchrotron sidebands. If all synchrotron sidebands overlap with the primary spin resonance, these sidebands can be summed to give rise to the primary spin resonance with a phase shift [5].

\subsection{Detuning Parameter in the AGS}

The bunched beam manipulation requires detuning parameter $\alpha_{z z}$ to ensure that SFPs of the 1:1 parametric resonance remain inside the dynamical aperture. The detuning parameter arises mainly from sextupole and octupole fields. The bottom plot of Fig. 4 shows the measured chromaticities as a function of the beam momentum in $(\mathrm{GeV} / \mathrm{c})$ [11]. Solid lines are chromaticities obtained from a fit with the integrated sextupole field strength

$$
\begin{aligned}
& S_{b}=-0.0003875+0.03228 / p-\left(0.00008 p+0.000001 p^{2}+0.0000009 p^{3}\right)\left(\mathrm{m}^{-1}\right) \\
& S_{e}=-0.01\left(\mathrm{~m}^{-1}\right)
\end{aligned}
$$

for short AGS bending magnets $(2.0066 \mathrm{~m})$. For long magnets $(2.3876 \mathrm{~m})$ in the AGS, the integrated sextupole strength of the $S_{b}$ term is assumed to be proportional to their length. Here $p$ is the beam momentum in $(\mathrm{GeV} / \mathrm{c}), S_{b}$ is the integrated sextupole field in each dipole distributed in the whole dipole, and $S_{e}$ is the integrated sextupole field distributed only at the end of each dipole. The first term in $S_{b}$ may be considered as the systematic error term. The eddy current contribution, which is inversely proportional to the beam momentum, depends on the $\dot{B}$. In this specific experiment [11], we have $\dot{B}=2 \mathrm{~T} / \mathrm{s}$. The saturation term is nonlinear with respect to the momentum $p$. The end sextupole in the $S_{e}$ term is assumed to be momentum independent. 
It is worth pointing out that the above sextupole model may not be fully justified by fitting only the data from the chromaticity measurements. Detailed beam measurements are needed to obtain a better model.

There are two family of chromatic sextupoles in the AGS. The vertical chromatic sextupoles are located at all 7 th straight sections. The horizontal chromatic sextupoles are located at the 13th straight sections except the C, F, I, and L superperiods. Using these two families of sextupoles, the chromaticities can be adjusted to zero easily. The resulting detuning parameters, $\alpha_{x x}, \alpha_{x z}$, and $\alpha_{z z}$ are shown on the top plot of Fig. 4. Since $\alpha_{z z}$ is less than zero for all momentum, the detuning parameters seen by the beam can be reinforced by choosing octupoles with negative detuning octupole field gradients. To minimize vertical tune spread

of the beam, it. is preferable to have a machine with zero chromaticity and zero $\alpha_{x z}$.

\section{Conclusion}

The method of overcoming intrinsic spin resonances by using the 1:1 parametric resonance island created by rf dipoles is studied. Analytic formula and numerical simulations are used to evaluate the feasibility and difficulties of this method. We find that the beam should be adiabatically transferred to the outer parametric resonance island. The chromaticity should be corrected to minimize the betatron tune spread vs the particle momentum. The required betatron amplitude detuning parameter can be generated by octupoles. Using the realistic AGS lattice, the method can indeed be used to provide polarized beam acceleration through intrinsic resonances. Experimental tests should be carried out in order to study the feasibility of the method present in this study. 


\section{Appendix}

\section{A Detuning coefficients}

The particle Hamiltonian in the presence of octupole magnets is given by

$$
H=\frac{1}{2}\left(x^{\prime 2}+z^{\prime 2}\right)+\frac{1}{2}\left(K_{x}(s) x^{2}+K_{z}(s) z^{2}\right)+\frac{1}{24} \frac{B^{\prime \prime \prime}(s)}{B \rho}\left(x^{4}-6 x^{2} z^{2}+z^{4}\right),
$$

where $K_{x}$ and $K_{z}$ are focusing functions, $B^{\prime \prime \prime}(s)=\left.B^{\prime \prime \prime} \Delta L\right|_{i} \delta\left(s-s_{i}\right)$ is the strength function of the $i$-th octupole magnet. Here $\left.B^{\prime \prime \prime} \Delta L\right|_{i}$ is the corresponding integrated octupole strength. Using the Floquet transformation with

$$
x=\sqrt{2 \beta_{x} J_{x}} \cos \left(\psi_{x}\right), \quad z=\sqrt{2 \beta_{z} J_{z}} \cos \left(\psi_{z}\right),
$$

and using the angle $\theta=s / R$ as the time variable, the nonlinear detuning to the Hamiltonian is given by

$$
H=\nu_{x} J_{x}+\nu_{z} J_{z}+\frac{1}{2} \alpha_{x x} J_{x}^{2}+\alpha_{x z} J_{x} J_{z}+\frac{1}{2} \alpha_{z z} J_{z}^{2}+\cdots
$$

where

$$
\begin{aligned}
& \alpha_{z z}=\frac{\beta_{z}^{2}}{16 \pi B \rho} \sum_{i}\left(B^{\prime \prime \prime} \Delta L\right)_{i}, \\
& \alpha_{x z}=-\frac{\beta_{z} \beta_{x}}{8 \pi B \rho} \sum_{i}\left(B^{\prime \prime \prime} \Delta L\right)_{i}, \quad \alpha_{x x}=\frac{\beta_{x}^{2}}{16 \pi B \rho} \sum_{i}\left(B^{\prime \prime \prime} \Delta L\right)_{i} .
\end{aligned}
$$

Besides the nonlinear detuning, octupoles generate also half-integer and 4 th order nonlinear resonances. The AGS lattice is made of 12 superperiods with 5 nearly identical FODO cells per superperiod. It has a weak 60 FODO periods. Since the betatron tunes are about 8.8 , the "best" octupole arrangement is a configuration with 4,6 or 12 octupoles located symmetrically with respect to the AGS machine. Let $N$ be the number of octupoles, i.e. $N=4,6$ or 12. The driving terms for the $2 \nu_{x}-2 \nu_{z}=0$ and $4 \nu_{z}=36$ resonances are respectively given by

$$
\begin{aligned}
& H_{2,-2 ; 0}=-\left\{\frac{1}{16 \pi B \rho} N\left(B^{\prime \prime \prime} \Delta L\right)_{i} \beta_{x} \beta_{x}\right\} J_{x} J_{z} \cos \left(2 \phi_{x}-2 \phi_{z}\right) \\
& H_{0,4 ; 36}=-\left\{\frac{1}{96 \pi B \rho} N\left(B^{\prime \prime \prime} \Delta L\right)_{i} \beta_{x}^{2}\right\} J_{z}^{2} \cos \left(4 \phi_{z}-36 \theta\right) .
\end{aligned}
$$

The effect of these resonances can be minimized by moving the betatron tunes away from these resonance lines.

Acknowledgment

We thank Ed. Bleser, C. Gardner, and L. Ahrens for helpful discussions on the AGS modeling. 


\section{References}

[1] L.H. Thomas, Phil. Mag. $\underline{3}, 1$ (1927); V.Bargmann, L. Michel, and V.L. Telegdi, Phys. Rev. Lett. 2, 435 (1959).

[2] E.D. Courant and R. Ruth, BNL report, BNL-51270 (1980).

[3] S. Tepikian, S.Y. Lee, and E.D. Courant, Particle Accelerators, 20, 1 (1986).

[4] H. Huang et al., Preservation of polarization with a partial snake, Phys. Rev. Lett. 73, 2982 (1994), Ph.D. Thesis, Indiana University (1994).

[5] S.Y. Lee and M. Berglund, Phys. Rev. E53, (1996).

[6] F.Z. Khiari et al., Phys. Rev. D39, 45 (1989); Ph.D. Thesis, University of Michigan, (1988).

[7] L.A. Ahrens, AIP Conference Proceedings, No.187, K. Heller, ed., p. 1068 (AIP, N.Y. 1988).

[8] M. Froissart, and R. Stora, Nucl. Inst. Meth. 7, 297 (1960).

[9] H. Huang et al., Phys. Rev. E48, 4678 (1993); M. Ellison et al., Phys. Rev. Lett. 70, 591 (1993); M. Syphers et al., Phys. Rev. Lett. 71, 719 (1993); Y. Wang et al., Phys. Rev. E 49, 1610 (1994).

[10] S.Y. Lee, Phys. Rev. E49, 5706 (1994).

[11] E. Bleser, AGS Tech-note No. 288 (1987); E. Auerbach, E. Bleser, R. Thern, AGS Tech-Note No. 276 (1987). 


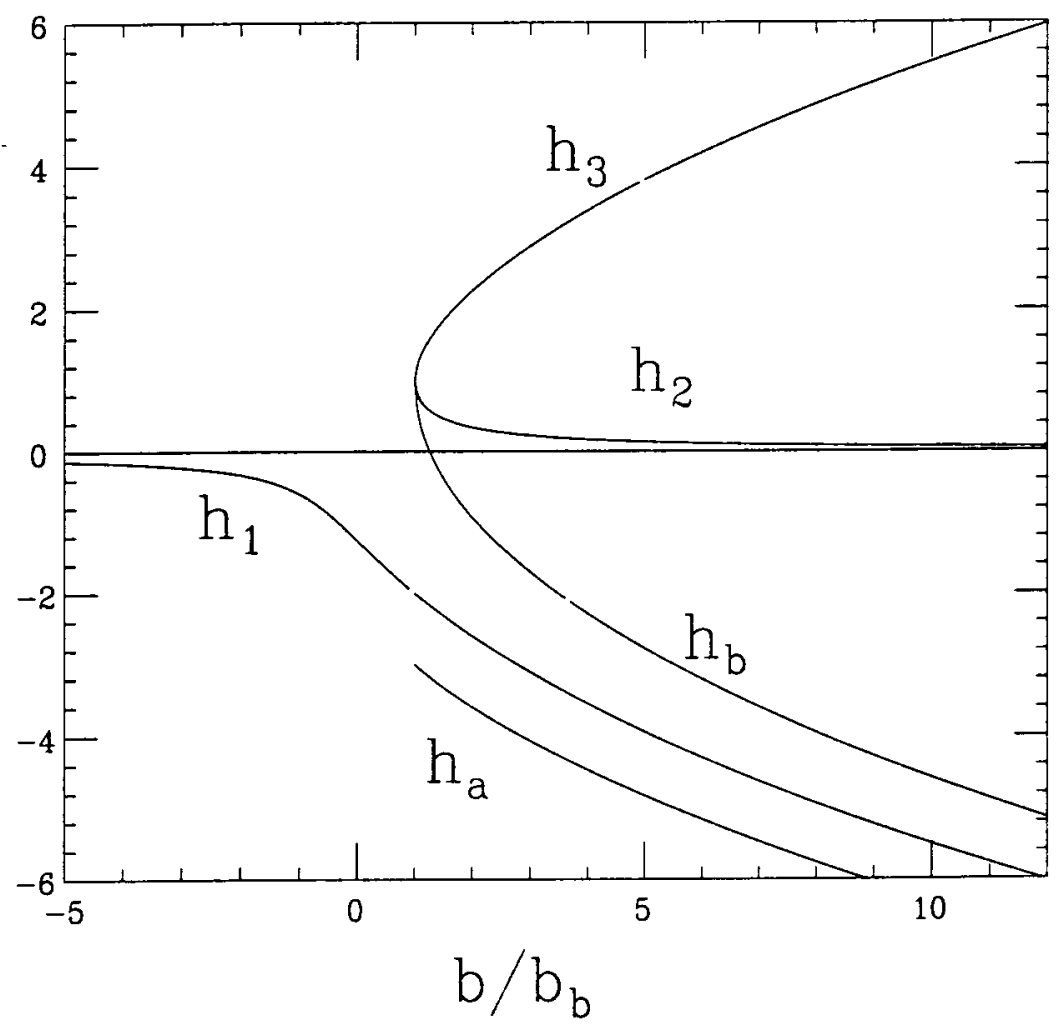

Figure 1: The fixed points, in the normalized coordinate, of the 1:1 parametric resonance Hamiltonian is plotted as a function of the modulation frequency parameter $x=b / b_{b}$. 

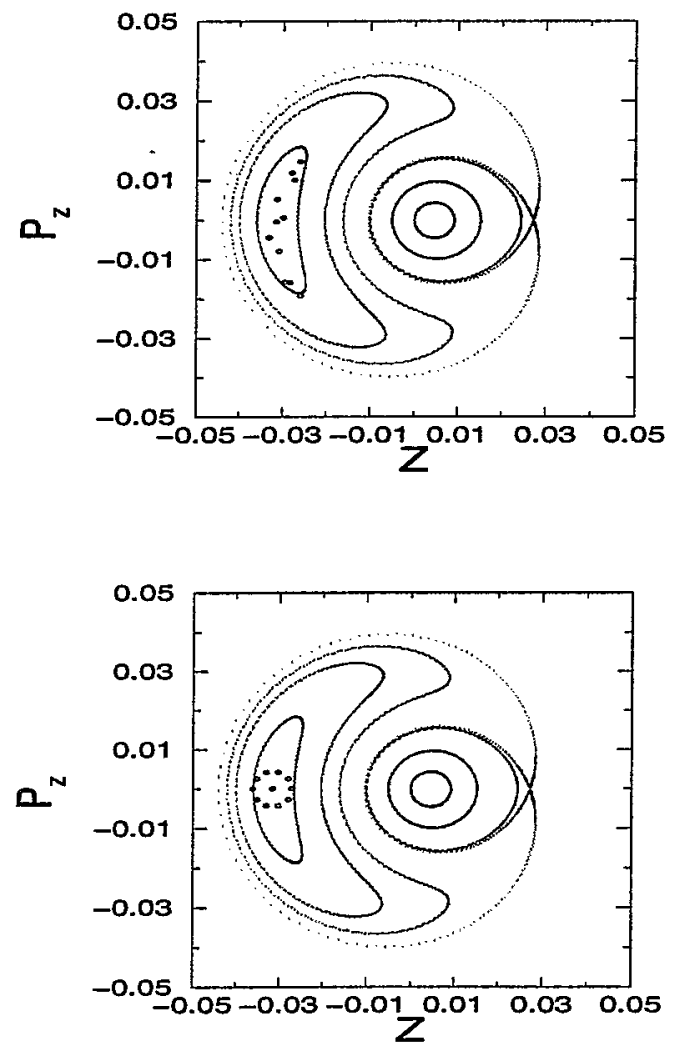

Figure 2: The bottom plot shows the beam bunch with a $95 \%$ normalized emittance of $20 \pi \mathrm{mm}$-mrad being kicked into the outer island of the 1:1 parametric resonance. The top plot shows the evolution of the ellipse after 500 orbital revolutions. The mismatch of the Hamiltonian contour can cause a substantial emittance dilution. 

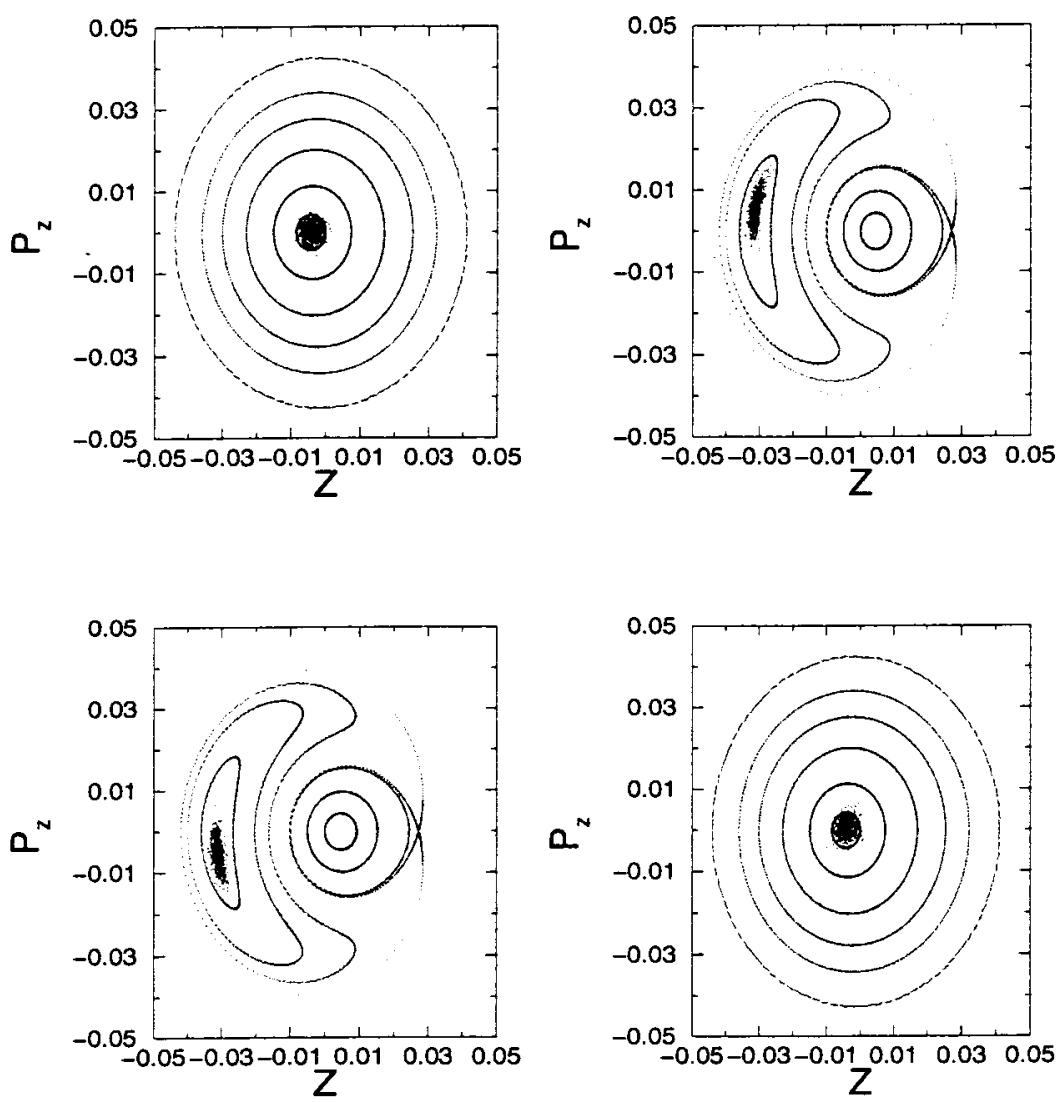

Figure 3: The top-left plot shows the beam distribution in the Hamiltonian contour. The top-right plot shows the beam profile and the Hamiltonian contour after the betatron tune is adiabatically ramped by 0.02 in 600 revolutions. The bottom-left plot shows the beam profile in the Poincare surface of section when the betatron tune is stationed for the spin resonance crossing. The bottom-right plot shows the beam profile after the betatron tune is adiabatically restored to its original value. Note that the emittance is preserved in these beam manipulations. 


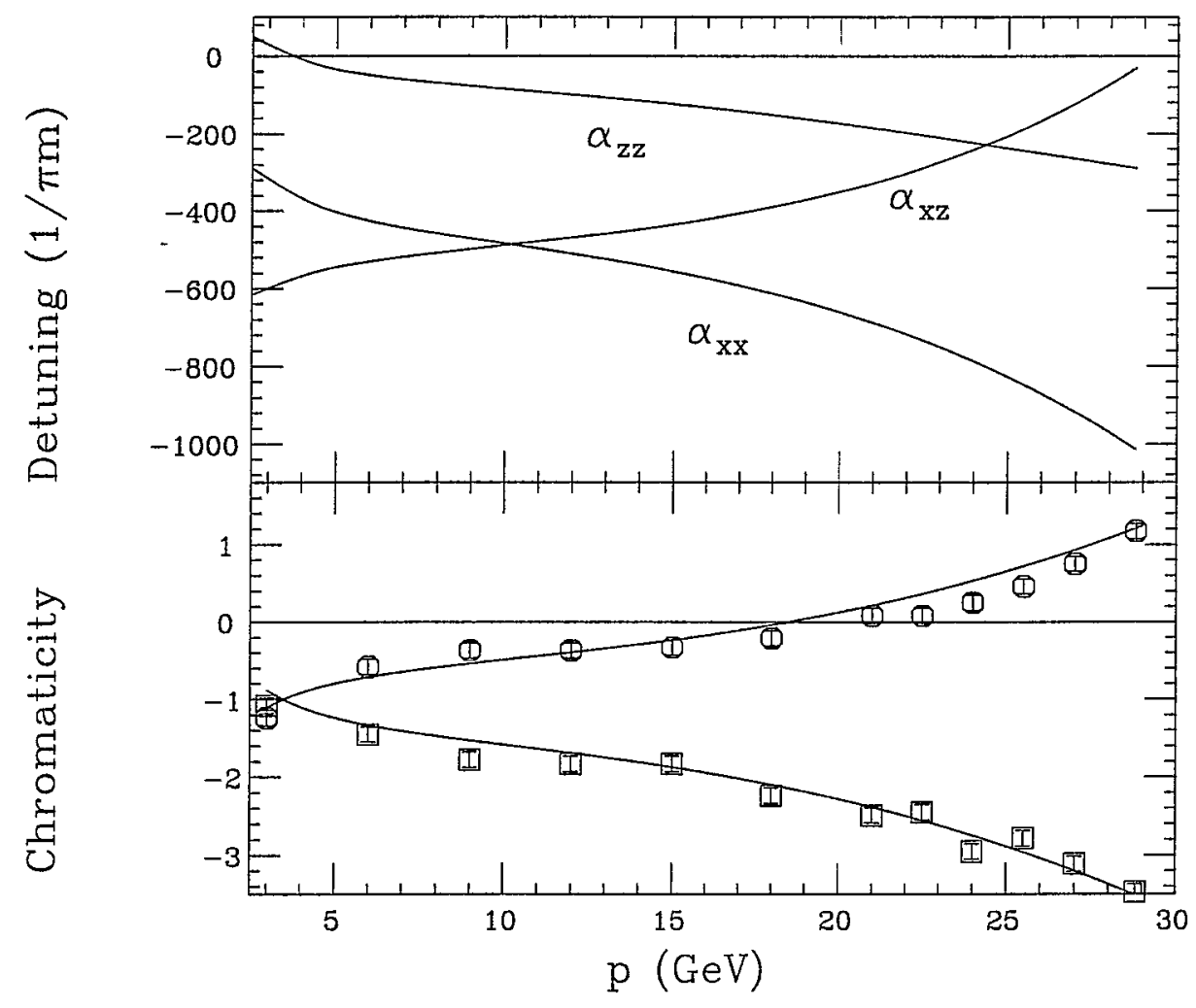

Figure 4: The bottom plot shows the measured chromaticities of the AGS [11] as a function of the beam momentum. This set of data is used to deduce the intrinsic sextupole field in A the AGS Ring. The top plot shows the nonlinear detuning parameters when the chromaticity of the AGS is corrected to zero with two families of chromatic sextupoles. 


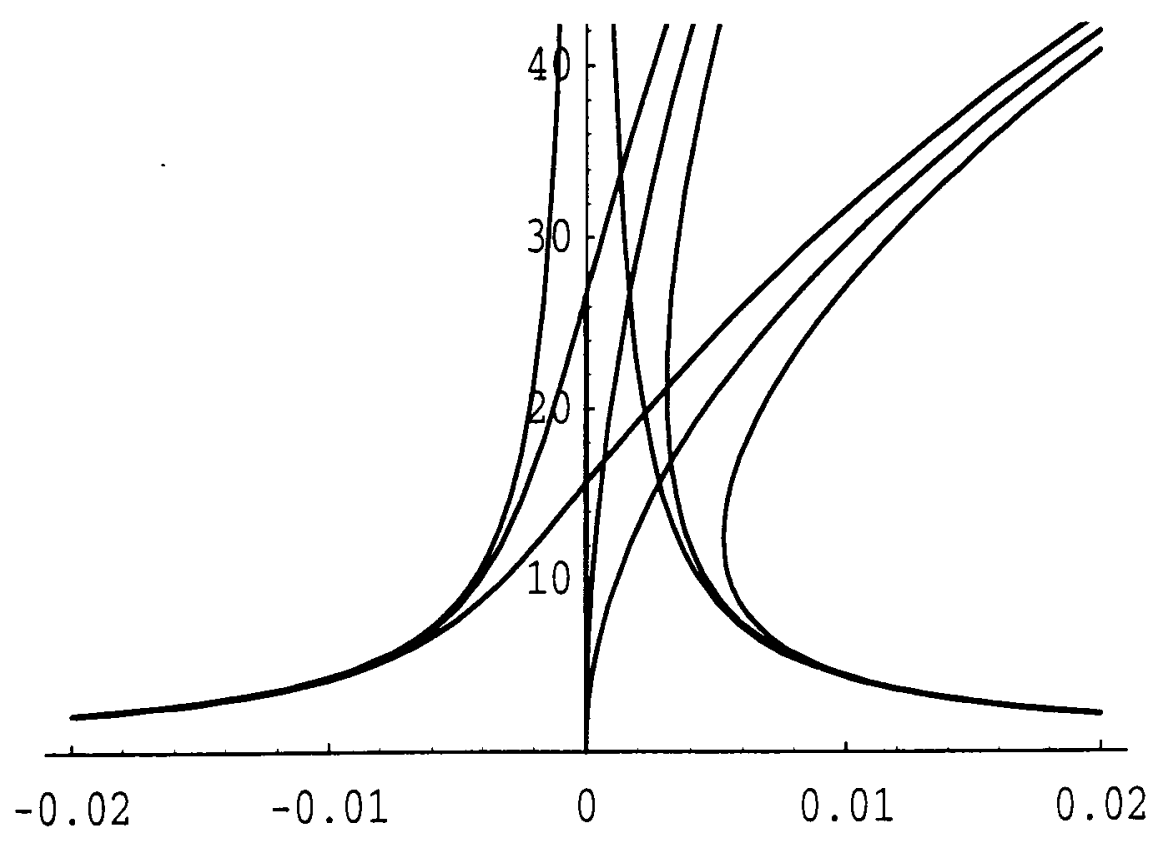

Figure 5: The distance (in $\mathrm{mm}$ ) of the fix points from the reference orbit is shown as a function of the proximity parameter $\delta$. The three set of curves show the fix point locations and nonlinear detuning curve with detuning parameter of 0,100 , and $500 \mathrm{~m}^{-1}$ from left to right, respectively. 